\title{
A PRIORI GENERALIZATION ERROR ANALYSIS OF TWO-LAYER NEURAL NETWORKS FOR SOLVING HIGH DIMENSIONAL SCHRÖDINGER EIGENVALUE PROBLEMS
}

\author{
JIANFENG LU AND YULONG LU
}

\begin{abstract}
This paper analyzes the generalization error of two-layer neural networks for computing the ground state of the Schrödinger operator on a $d$-dimensional hypercube. We prove that the convergence rate of the generalization error is independent of the dimension $d$, under the a priori assumption that the ground state lies in a spectral Barron space. We verify such assumption by proving a new regularity estimate for the ground state in the spectral Barron space. The later is achieved by a fixed point argument based on the Krein-Rutman theorem.
\end{abstract}

\section{INTRODUCTION}

High dimensional partial differential equations (PDEs) arise ubiquitously from scientific and engineering problems which involve many degrees of freedom, examples include many-body quantum mechanics, phase space description of chemical dynamics, learning and control of complex systems, spectral methods for high dimensional data, just to name a few. While numerical methods for partial differential equations in low-dimension are quite standard, the numerical solution to high dimensional PDEs has remained an outstanding challenge due to the well-known curse of dimensionality. Namely, the computational cost can grow exponentially as the dimension increases. Perhaps the most celebrated and important example of such challenge is to determine the ground state of many-body quantum systems, which amounts to solving eigenvalue problems for high dimensional PDEs.

In recent years, the artificial neural-networks have shown great success in representing high-dimensional classifiers or probability distributions in a variety of machine learning tasks and have led to the tremendous success and popularity of deep learning [23, 29]. Motivated by those recent success, researchers have been actively exploring using deep learning techniques to solve high dimensional PDEs [7, 12, 15, 16, 28, 35, 38] by parametrizing the solution as a neural network, including eigenvalue problems for many-body quantum systems (see e.g., [5, 6, 8, 13, 17 19, 27]). Despite wide popularity and many successful examples of employing neural network ansatz for solving PDEs, their theoretical analyses are still sparse. For the recent theoretical development, we refer the interested readers to [20, 25, 26, 31, 32, 37]. Nonetheless, to the best of our knowledge, the numerical analysis of neural network methods for high dimensional eigenvalue problems is not yet established. The goal of this paper is to provide an a priori generalization

J.L. is supported in part by National Science Foundation via grants DMS-2012286 and CCF1934964. Y.L. is supported by the start-up fund of the Department of Mathematics and Statistics at UMass Amherst. 
analysis of variational methods for computing the ground state of the Schrödinger operator in high dimension based on the two-layer neural network ansatz.

Our generalization error analysis follows largely the framework established in our previous work [24], where the a priori generalization error is analyzed for deep Ritz method for solving elliptic equations. In particular, to establish approximation results that do not deteriorate as dimension increases, we will work in the spectral Barron space [3, 10, 24, 34]. In fact, it has been shown in those works that Barron functions has "lower complexity" than more familiar regularity-based functions such as Sobolev or Hölder functions in the sense that the former can be efficiently approximated by two-layer neural networks without curse of dimensionality. On the other hand, as the Barron space is rather different from Sobolev or Hölder spaces, the main challenge one faces is to establish regularity theory for high dimensional PDEs in such space. Our previous work [24] established the appropriate solution theory for elliptic equations. The key contribution of the present work is to extend such novel solution theory to Schrödinger eigenvalue problems in high dimension. Since we are working in spectral Barron space, which is a general Banach space without inner product structure, Lax-Milgram or Courant-Fisher theorems are not applicable, and thus we have to rely on fixed point theorem to establish the existence of solutions. In our previous work for elliptic PDEs [24], Fredholm alternative principle was used; while in this work, to establish the existence of nontrivial eigenfunctions, we rely on the Krein-Rutman theorem [22].

The remainder of this paper is organized as follows. In Section 2 we first set up the ground state problem of the Schrödinger operator and present the main generalization results (see Theorems 2.3.2.4) and the new regularity estimate on the ground state in the spectral Barron space (see Theorem 2.5). In Section 3 we prove a key stability estimate on the ground state, which allows us to bound the $H^{1}$-error between the ground state and its approximation in terms of the energy excess. We present the proof of the main generalization result in Section 4 and the proof of the new regularity estimate on the ground state in Section 5 .

\section{Set-Up and Main Results}

2.1. Set-Up of Problem. Let $\Omega=[0,1]^{d}$ be the unit hypercube on $\mathbb{R}^{d}$ with the boundary $\partial \Omega$. Consider the Neumann eigenvalue problem for the Schrödinger operator

$$
\begin{array}{lrl}
\mathcal{H} u=-\Delta u+V u=\lambda u & \text { in } \Omega, \\
\frac{\partial u}{\partial \nu}=0 & \text { on } \partial \Omega .
\end{array}
$$

where $\mathcal{H}:=-\Delta+V$ is the Schrödinger operator with the potential function $V$ equipped with the Neumann boundary condition. We are particularly interested in computing the ground state of $\mathcal{H}$, that is the eigenfunction associated to the smallest eigenvalue of $\mathcal{H}$.

Throughout the paper we make the following minimum assumption on the potential function.

Assumption 2.1. There exist finite positive constants $V_{\min }$ and $V_{\max }$ such that $V_{\min } \leq V(x) \leq V_{\max }$ for every $x \in \Omega$.

Note that we do not lose generality by assumption that $V_{\min }$ is positive, since one can always add a constant to $V$ without changing the eigenfunctions. 
It is well-known that the minimum eigenvalue $\lambda_{0}$ can be characterized as the minimum of Rayleigh quotient, i.e.

$$
\lambda_{0}=\min _{u \in H^{1}(\Omega)} \mathcal{E}(u):=\min _{u \in H^{1}(\Omega)} \frac{\langle u, \mathcal{H} u\rangle}{\langle u, u\rangle},
$$

where $\langle\cdot, \cdot\rangle$ denotes the inner product on $L^{2}(\Omega)$. Under Assumption 2.1, the minimizer of the variational problem (2.1) is achieved at the ground state $u_{0} \in H^{1}(\Omega)$. Moreover, the ground state $u_{0}$ is unique (up to a multiplicative constant) and is strictly positive (up to a global sign) on $\Omega$; see e.g., [14, Theorem 3.3.2]. Without loss of generality we assume further that the ground state $u_{0}$ is normalized, i.e., $\left\|u_{0}\right\|_{L^{2}(\Omega)}=1$.

For certain results to hold, we may also need to make the following additional spectrum assumption on the Schrödinger operator $\mathcal{H}$.

Assumption 2.2. The operator $\mathcal{H}$ has discrete spectrum $\left\{\lambda_{j}\right\}_{j=0}^{\infty}$ with a positive spectral gap, i.e. $\lambda_{0}<\lambda_{1} \leq \lambda_{2} \leq \cdots \leq \lambda_{k} \uparrow \infty$.

To avoid any confusion with the subscript in the notation, let us denote the ground state eigenpair by $\left(\lambda^{*}, u^{*}\right):=\left(\lambda_{0}, u_{0}\right)$, which our study focuses on.

The natural idea is to seek an approximate solution to Problem (2.1) within some hypothesis class $\mathcal{F} \subset H^{1}(\Omega)$ that are parameterized by neural networks. In practice, the Monte-Carlo method is employed to compute the high dimensional integrals defined by the inner products in (2.1), leading to the definition of empirical loss (or risk) minimization. More concretely, let us denote by $\mathcal{P}_{\Omega}$ the uniform probability distribution on the domain $\Omega$. Then the population loss $\mathcal{E}$ can be written as

$$
\mathcal{E}(u)=\frac{\mathcal{E}_{V}(u)}{\mathcal{E}_{2}(u)}:=\frac{\mathbf{E}_{X \sim \mathcal{P}_{\Omega}}\left[|\nabla u(X)|^{2}+V(X)|u(X)|^{2}\right]}{\mathbf{E}_{X \sim \mathcal{P}_{\Omega}}\left[|u(X)|^{2}\right]} .
$$

Let $\left\{X_{j}\right\}_{j=1}^{n}$ be a sequence of random variables that are independent and identically distributed (i.i.d.) according to $\mathcal{P}_{\Omega}$. The population loss is approximated by the following empirical loss

$$
\mathcal{E}_{n}(u)=\frac{\mathcal{E}_{n, V}(u)}{\mathcal{E}_{n, 2}(u)},
$$

where $\mathcal{E}_{n, V}$ and $\mathcal{E}_{n, 2}$ are defined by

$$
\begin{aligned}
\mathcal{E}_{n, V} & :=\frac{1}{n} \sum_{j=1}^{n}\left(\left|\nabla u\left(X_{j}\right)\right|^{2}+V\left(X_{j}\right)\left|u\left(X_{j}\right)\right|^{2}\right) \\
\mathcal{E}_{n, 2} & :=\frac{1}{n} \sum_{j=1}^{n}\left|u\left(X_{j}\right)\right|^{2} .
\end{aligned}
$$

Note that we have used the fact that $|\Omega|=1$ in deriving the Monte-Carlo approximation above. Let $u_{n}$ be a minimizer of $\mathcal{E}_{n}$ within $\mathcal{F}$, i.e., $u_{n}=\arg \min _{u \in \mathcal{F}} \mathcal{E}_{n}(u)$. Again since $\mathcal{E}_{n}(u)$ is scaling-invariant, we may assume that $\left\|u_{n}\right\|_{L^{2}}=1$. Our goal is to obtain quantitative estimates for the error between $u_{n}$ and $u^{*}$, following the statistical learning literature, we will call such error the generalization error.

We are interested in quantifying the error between $u_{n}$ and $u^{*}$ in terms of two quantities. The first one is given by the energy excess $\mathcal{E}\left(u_{n}\right)-\mathcal{E}\left(u^{*}\right)$ that quantifies the approximation of $\mathcal{E}\left(u_{n}^{m}\right)$ to the leading eigenvalue $\lambda^{*}=\mathcal{E}\left(u^{*}\right)$. 
To introduce the second quantity for measuring the error, we define the projection operator $P$ onto the space of ground state by setting

$$
P u=\left\langle u, u^{*}\right\rangle u^{*} \text {. }
$$

Let us also define the operator $P^{\perp}=(I-P)$, i.e.

$$
P^{\perp} u=u-\left\langle u, u^{*}\right\rangle u^{*}
$$

Notice that if both $u_{0}$ and $u$ are normalized, then

$$
\left\|P^{\perp} u\right\|_{L^{2}}^{2}=1-\left|\left\langle u, u^{*}\right\rangle\right|^{2} .
$$

Therefore $\left\|P^{\perp} u_{n}\right\|_{L^{2}(\Omega)}$ quantifies the offset of the direction of $u_{n}$ from that of $u^{*}$. The following theorem shows that the $H^{1}$-norm of $P^{\perp} u$ can be bounded above by the energy excess.

Proposition 2.1. Assume that $\mathcal{H}$ satisfies Assumptions 2.1 and 2.2. Then for any $u \in H^{1}(\Omega)$,

$$
\begin{aligned}
& \left\|P^{\perp} u\right\|_{L^{2}(\Omega)}^{2} \leq \frac{\mathcal{E}(u)-\mathcal{E}\left(u^{*}\right)}{\lambda_{1}-\lambda^{*}}\|u\|_{L^{2}(\Omega)}^{2}, \\
& \left\|\nabla P^{\perp} u\right\|_{L^{2}(\Omega)}^{2} \leq\left(\mathcal{E}(u)-\mathcal{E}\left(u^{*}\right)\right)\left(\frac{V_{\max }-V_{\min }}{\lambda_{1}-\lambda^{*}}+1\right)\|u\|_{L^{2}(\Omega)}^{2} .
\end{aligned}
$$

In particular, if $\|u\|_{L^{2}}=1$, then

$$
\left\|P^{\perp} u\right\|_{H^{1}(\Omega)}^{2} \leq\left(\frac{V_{\max }-V_{\min }+1}{\lambda_{1}-\lambda^{*}}+1\right)\left(\mathcal{E}(u)-\mathcal{E}\left(u^{*}\right)\right) .
$$

2.2. Main Results. We aim to prove quantitative generalization error estimates between the approximation ground state $u_{n}$ parametrized by neural networks and the exact ground state $u^{*}$. Our particular interest is to show that under certain circumstances the generalization error of the neural network solution does not suffer from the curse of dimensionality. To this end, we will assume (and prove below) that the exact ground state $u^{*}$ lies in a smaller function space than the usual Sobolev space within which the functions can be approximated by neural networks without curse of dimensionality. Specifically, we assume that $u^{*}$ belongs to the spectral Barron space [24] defined as follows.

Let us first define the set of cosine functions

$$
\mathcal{C}=\left\{\Phi_{k}\right\}_{k \in \mathbb{N}_{0}^{d}}:=\left\{\prod_{i=1}^{d} \cos \left(\pi k_{i} x_{i}\right) \mid k_{i} \in \mathbb{N}_{0}\right\} .
$$

Let $\{\hat{u}(k)\}_{k \in \mathbb{N}_{0}^{d}}$ be the expansion coefficients of a function $u \in L^{1}(\Omega)$ under the basis $\left\{\Phi_{k}\right\}_{k \in \mathbb{N}_{0}^{d}}$. For $s \geq 0$, the spectral Barron space $\mathcal{B}^{s}(\Omega)$ on $\Omega$ is defined by

$$
\mathcal{B}^{s}(\Omega):=\left\{u \in L^{1}(\Omega): \sum_{k \in \mathbb{N}_{0}^{d}}\left(1+\pi^{s}|k|_{1}^{s}\right)|\hat{u}(k)|<\infty\right\},
$$

which is equipped with the spectral Barron norm

$$
\|u\|_{\mathcal{B}^{s}(\Omega)}=\sum_{k \in \mathbb{N}_{0}^{d}}\left(1+\pi^{s}|k|_{1}^{s}\right)|\hat{u}(k)| .
$$

Note that we use $|k|_{1}$ to denote the $\ell^{1}$-norm of a vector $k$. It is clear that $B^{s}(\Omega)$ is a Banach space. Moreover, since functions in $B^{s}(\Omega)$ have summable cosine coefficients. we have $B^{s}(\Omega) \hookrightarrow C(\bar{\Omega})$. When $s=2$, we adopt the short notation $\mathcal{B}(\Omega)$ 
for $\mathcal{B}^{2}(\Omega)$. Our notion of spectral Barron space is an adaption of the Barron space defined in the seminal work [3]; see also the recent works [2, 11, 21, 33] on other variants of Barron spaces. The original Barron function $f$ in [3] is defined on the whole space $\mathbb{R}^{d}$ whose Fourier transform $\hat{f}(w)$ satisfies that $\int|\hat{f}(\omega)||\omega| d \omega<\infty$. Our spectral Barron space $\mathcal{B}^{s}(\Omega)$ with $s=1$, defined on the bounded domain $\Omega$, can be viewed as a finite domain analog of the original Barron space from [3].

Functions in the spectral Barron space differ substantially from those in Sobolev or Hölder spaces; most importantly, they can be approximated with respect to the $H^{1}$-norm by two-layer neural networks without curse of dimensionality. To make this more precise, we recall an approximation result from [24]. Let us define for an activation function $\phi$, a constant $B>0$ and the number of hidden neurons $m$ the set of functions

$$
\mathcal{F}_{\phi, m}(B):=\left\{c+\sum_{i=1}^{m} \gamma_{i} \phi\left(w_{i} \cdot x-t_{i}\right),|c| \leq 2 B,\left|w_{i}\right|_{1}=1,\left|t_{i}\right| \leq 1, \sum_{i=1}^{m}\left|\gamma_{i}\right| \leq 4 B\right\} .
$$

We will focus on the rescaled Softplus activation function

$$
\mathrm{SP}_{\tau}(z)=\frac{1}{\tau} \mathrm{SP}(\tau z)=\frac{1}{\tau} \ln \left(1+e^{\tau z}\right)
$$

where $\tau>0$ is a rescaling parameter. Observe that $\mathrm{SP}_{\tau} \rightarrow \operatorname{ReLU}$ pointwisely as $\tau \rightarrow 0$ (see [24, Lemma 4.6]).

Let $\mathcal{F}_{\mathrm{SP}_{\tau}, m}(B)$ be the set of neural networks defined by setting $\phi=\mathrm{SP}_{\tau}$ in (2.5). The following lemma shows that functions in $\mathcal{B}(\Omega)$ can be well approximated by functions in $\mathcal{F}_{\mathrm{SP}_{\tau}, m}(B)$ without curse of dimensionality.

Lemma 2.2. [24, Theorem 2.2] For any $u \in \mathcal{B}(\Omega)$, there exists a two-layer neural network $u_{m} \in \mathcal{F}_{\mathrm{SP}_{\tau}, m}\left(\|u\|_{\mathcal{B}(\Omega)}\right)$ with $\tau=\sqrt{m}$, such that

$$
\left\|u-u_{m}\right\|_{H^{1}(\Omega)} \leq \frac{\|u\|_{\mathcal{B}(\Omega)}(6 \log m+30)}{\sqrt{m}} .
$$

With the above approximation result at hand, we are ready to state the main generalization theorem as follows.

Theorem 2.3. Assume that $\mathcal{H}$ satisfies Assumptions [2.1] and 2.2. Assume also that the ground state $u^{*} \in \mathcal{B}(\Omega)$. Let $u_{n}^{m}$ be a minimizer of the empirical loss $\mathcal{E}_{n}$ within the set $\mathcal{F}=\mathcal{F}_{\mathrm{SP} \tau, m}(B)$ with $B=\left\|u^{*}\right\|_{\mathcal{B}(\Omega)}$ and with $\tau=\sqrt{m}$. Given $\delta \in\left(0, \frac{1}{3}\right)$, assume that $n$ and $m$ are large enough so that $\xi_{i}(n, \delta) \leq 1 / 2, i=1,2,3$ and $\eta(B, m) \leq 1 / 2$ where $\xi_{i}(n, \delta)$ are defined in (4.3), (4.7) and (4.12), and $\eta(B, m)$ is defined in (4.16). Then with probability at least $1-3 \delta$,

$$
\mathcal{E}\left(u_{n}^{m}\right)-\mathcal{E}\left(u^{*}\right) \leq \frac{C_{1} \ln \left(\frac{1}{\delta}\right) \sqrt{m(\ln m+1)}}{\sqrt{n}}+\frac{C_{2}(\ln m+1)}{\sqrt{m}},
$$

where $C_{1}$ depends on $\left\|u^{*}\right\|_{\mathcal{B}(\Omega)}, d, V_{\max }$ polynomially and $C_{2}$ depends on $\left\|u^{*}\right\|_{\mathcal{B}(\Omega)}$ linearly. In particular, with the choice $m=\sqrt{n}$, we have that there exists $C_{3}>0$ such that with probability at least $1-3 \delta$,

$$
\mathcal{E}\left(u_{n}^{m}\right)-\mathcal{E}\left(u^{*}\right) \leq C_{3} \ln \left(\frac{1}{\delta}\right) \cdot n^{-\frac{1}{4}} .
$$

The proof of Theorem 2.3 relies on decomposing the generalization error into the sum of the approximation error (see Section 4.2) and statistical error arising 
from the Monte-Carlo approximation. The statistical error is further bounded by controlling the Rademacher complexity of certain neural network classes associated to the loss formulation (see Section 4.3). Thanks to Proposition 2.1. the generalization error in terms of the energy excess translates directly to that in terms of the $H^{1}$-norm of $P^{\perp} u_{n}^{m}$.

Theorem 2.4. Suppose that the assumption of Theorem 2.3 holds and suppose further that $\mathcal{H}$ has a spectral gap. Then there exist positive constants $C_{4}$ and $C_{5}$ depending polynomially on $\left\|u^{*}\right\|_{\mathcal{B}(\Omega)}, d, V_{\min }, V_{\max }$ and $\lambda_{1}-\lambda_{0}$ such that with probability at least $1-3 \delta$,

$$
\left\|P^{\perp} u_{n}^{m}\right\|_{H^{1}(\Omega)}^{2} \leq \frac{C_{4} \ln \left(\frac{1}{\delta}\right) \sqrt{m(\ln m+1)}}{\sqrt{n}}+\frac{C_{5} \ln \left(\frac{1}{\delta}\right)(\ln m+1)}{\sqrt{m}} .
$$

Setting $m=\sqrt{n}$ in the above leads to that the follow holds for some $C_{6}>0$ with probability at least $1-3 \delta$ :

$$
\left\|P^{\perp} u_{n}^{m}\right\|_{H^{1}(\Omega)}^{2} \leq C_{6} \ln \left(\frac{1}{\delta}\right) \cdot n^{-\frac{1}{4}} .
$$

Theorem 2.3 and Theorem 2.4 show that with high probability the convergence rate of the generalization error of two-layer network for approximating the ground state $u^{*}$ and the corresponding leading eigenvalue $\lambda^{*}=\mathcal{E}\left(u^{*}\right)$ does not suffer from the curse of dimensionality provided that the ground state $u^{*} \in \mathcal{B}(\Omega)$.

Finally we justify the regularity assumption on the ground state in the following theorem. This gives a novel solution theory of high dimensional eigenvalue problems in Barron type spaces.

Theorem 2.5. Assume that $V \in \mathcal{B}^{s}(\Omega)$ with $s \geq 0$ and $V$ satisfies Assumption 2.1. Then the ground state $u^{*} \in \mathcal{B}^{s+2}(\Omega)$.

Our idea of proving Theorem 2.5 differs from the standard proof of Sobolev regularity of eigenfunctions, which usually relies on bootstrapping estimates on the weak derivative of the eigenfunctions. Instead, we prove Theorem 2.5 by reformulating the ground state problem as a fixed point problem on the spectral Barron space $\mathcal{B}^{s}(\Omega)$; the existence of a nontrivial fixed point is proved by employing the celebrated Krein-Rutman theorem [22]. See Section 5 for a complete proof.

\section{Stability estimate of the ground state (Proof of Proposition 2.1)}

In this section, we show that the offset $\left\|P^{\perp} u\right\|_{L^{2}(\Omega)}$ of any $u \in H^{1}(\Omega)$ can be bounded by the energy excess $\mathcal{E}(u)-\mathcal{E}\left(u^{*}\right)$.

Proof. Note that by the spectral gap assumption

$$
\mathcal{H} P^{\perp}-\lambda_{0} P^{\perp} \geq \lambda_{1}-\lambda_{0}>0 .
$$

Let us decompose

$$
u=P u+P^{\perp} u=: \alpha u_{0}+u_{\perp},
$$


where $\alpha=\left\langle u, u_{0}\right\rangle$ and $\left\langle u_{\perp}, u_{0}\right\rangle=0$. Substituting above into the Rayleigh quotient, we have

$$
\begin{aligned}
\mathcal{E}(u) & =\frac{|\alpha|^{2} \lambda_{0}+\left\langle u_{\perp}, \mathcal{H} u_{\perp}\right\rangle}{\|u\|^{2}} \\
& \geq \frac{\lambda_{0}\|u\|^{2}+\left(\lambda_{1}-\lambda_{0}\right)\left\|u_{\perp}\right\|^{2}}{\|u\|^{2}} \\
& =\lambda_{0}+\left(\lambda_{1}-\lambda_{0}\right) \frac{\left\|u_{\perp}\right\|^{2}}{\|u\|^{2}} \\
& =\mathcal{E}\left(u^{*}\right)+\left(\lambda_{1}-\lambda_{0}\right) \frac{\left\|u_{\perp}\right\|^{2}}{\|u\|^{2}} .
\end{aligned}
$$

Thus, the $L^{2}$-norm of $u_{\perp} \equiv P^{\perp} u$ can be bounded as

$$
\left\|u_{\perp}\right\|^{2} \leq \frac{\mathcal{E}(u)-\mathcal{E}\left(u_{0}\right)}{\lambda_{1}-\lambda_{0}}\|u\|^{2} .
$$

Note that this bound cannot be improved as can be seen by taking a linear combination of the first and second eigenstates of $\mathcal{H}$.

To obtain the bound on $\nabla u^{\perp}$, we notice that

$$
\mathcal{E}(u)=\lambda_{0}+\frac{\left\langle u_{\perp},\left(\mathcal{H}-\lambda_{0}\right) u_{\perp}\right\rangle}{\|u\|^{2}}
$$

Thus

$$
\begin{aligned}
\left\langle u_{\perp},\left(\mathcal{H}-\lambda_{0}\right) u_{\perp}\right\rangle & =\left\|\nabla u_{\perp}\right\|_{L^{2}}^{2}+\left\langle u_{\perp},\left(V-\lambda_{0}\right) u_{\perp}\right\rangle \\
& \leq\left(\mathcal{E}(u)-\mathcal{E}\left(u_{0}\right)\right)\|u\|^{2} .
\end{aligned}
$$

Rearranging the terms, we arrive at

$$
\begin{aligned}
\left\|\nabla u_{\perp}\right\|^{2} & \leq-\left\langle u_{\perp},\left(V-\lambda_{0}\right) u_{\perp}\right\rangle+\left(\mathcal{E}(u)-\mathcal{E}\left(u_{0}\right)\right)\|u\|^{2} \\
& \leq\left(V_{\max }-V_{\min }\right)\left\|u_{\perp}\right\|^{2}+\left(\mathcal{E}(u)-\mathcal{E}\left(u_{0}\right)\right)\|u\|^{2} \\
& \stackrel{3.1}{\leq}\left(\mathcal{E}(u)-\mathcal{E}\left(u_{0}\right)\right)\left(\frac{V_{\max }-V_{\min }}{\lambda_{1}-\lambda_{0}}+1\right)\|u\|^{2} .
\end{aligned}
$$

\section{Proof of Theorem 2.3}

4.1. Oracle Inequality for the Generalization Error. We first introduce an oracle inequality for the empirical loss that decomposes the generalization error into the sum of approximation error and statistical error. Recall the population loss $\mathcal{E}$ and the empirical loss $\mathcal{E}_{n}$ defined in (2.2) and (2.3) respectively. Consider the minimization of $\mathcal{E}_{n}$ in a function class $\mathcal{F}$ and we denote by $u_{n}$ a minimizer of $\mathcal{E}$ within $\mathcal{F}$, i.e. $u_{n}=\arg \min _{u \in \mathcal{F}} \mathcal{E}_{n}(u)$.

We aim to bound the energy excess $\Delta \mathcal{E}_{n}:=\mathcal{E}\left(u_{n}\right)-\mathcal{E}\left(u^{*}\right)$ where $u^{*}$ is the exact ground state. Let us first decompose $\Delta \mathcal{E}_{n}$ as follows:

$$
\Delta \mathcal{E}_{n}=\mathcal{E}\left(u_{n}\right)-\mathcal{E}_{n}\left(u_{n}\right)+\mathcal{E}_{n}\left(u_{n}\right)-\mathcal{E}_{n}\left(u_{\mathcal{F}}\right)+\mathcal{E}_{n}\left(u_{\mathcal{F}}\right)-\mathcal{E}\left(u_{\mathcal{F}}\right)+\mathcal{E}\left(u_{\mathcal{F}}\right)-\mathcal{E}\left(u^{*}\right)
$$

Here $u_{\mathcal{F}}=\arg \min _{u \in \mathcal{F}} \mathcal{E}(u)$. Note that $\mathcal{E}_{n}\left(u_{n}\right)-\mathcal{E}_{n}\left(u_{\mathcal{F}}\right) \leq 0$ since $u_{n}$ is the minimizer of $\mathcal{E}_{n}$. Therefore

$\Delta \mathcal{E}_{n} \leq\left(\mathcal{E}\left(u_{n}\right)-\mathcal{E}_{n}\left(u_{n}\right)\right)+\left(\mathcal{E}_{n}\left(u_{\mathcal{F}}\right)-\mathcal{E}\left(u_{\mathcal{F}}\right)\right)+\left(\mathcal{E}\left(u_{\mathcal{F}}\right)-\mathcal{E}\left(u^{*}\right)\right)=: T_{1}+T_{2}+T_{3}$.

Note that $T_{1}$ and $T_{2}$ are statistical error terms that arises from Monte-Carlo approximation for integration. The third term $T_{3}$ is the approximation error term due to restricting the minimization of $\mathcal{E}$ from over the set $H^{1}(\Omega)$ to $\mathcal{F}$; see an 
upper bound of $T_{3}$ in Theorem 4.5 when $\mathcal{F}$ is chosen as the set of two-layer neural networks. To control the statistical errors, we employ the well-known tool of Rademacher complexity, for which we recall its definition as follows.

Definition 4.1. We define for a set of random variables $\left\{Z_{j}\right\}_{j=1}^{n}$ independently distributed according to $\mathcal{P}_{\Omega}$ and a function class $\mathcal{S}$ the random variable

$$
\hat{R}_{n}(\mathcal{S}):=\mathbf{E}_{\sigma}\left[\sup _{g \in \mathcal{S}}\left|\frac{1}{n} \sum_{j=1}^{n} \sigma_{j} g\left(Z_{j}\right)\right| \mid Z_{1}, \cdots, Z_{n}\right],
$$

where the expectation $\mathbf{E}_{\sigma}$ is taken with respect to the independent uniform Bernoulli sequence $\left\{\sigma_{j}\right\}_{j=1}^{n}$ with $\sigma_{j} \in\{ \pm 1\}$. Then the Rademacher complexity of $\mathcal{S}$ defined by $R_{n}(\mathcal{S})=\mathbf{E}_{\mathcal{P}_{\Omega}}\left[\hat{R}_{n}(\mathcal{S})\right]$.

Now we are ready to bound $T_{1}$ and $T_{2}$ in terms of Rademacher complexities of suitable sets.

Bounding $T_{1}$. Thanks to the scaling-invariance of $\mathcal{E}$ and $\mathcal{E}_{n}$, we can assume that $u_{n}$ is normalized, i.e. $\left\|u_{n}\right\|_{2}=1$. Hence we have

$$
\begin{aligned}
T_{1} & \leq\left|\frac{\mathcal{E}_{n, V}\left(u_{n}\right)}{\mathcal{E}_{n, 2}\left(u_{n}\right)}-\frac{\mathcal{E}_{V}\left(u_{n}\right)}{\mathcal{E}_{2}\left(u_{n}\right)}\right| \\
& \leq \frac{\left|\mathcal{E}_{n, V}\left(u_{n}\right)-\mathcal{E}_{V}\left(u_{n}\right)\right|}{\mathcal{E}_{n, 2}\left(u_{n}\right)}+\frac{\mathcal{E}_{V}\left(u_{n}\right)}{\mathcal{E}_{2}\left(u_{n}\right) \cdot \mathcal{E}_{n, 2}\left(u_{n}\right)}\left|\mathcal{E}_{2}\left(u_{n}\right)-\mathcal{E}_{n, 2}\left(u_{n}\right)\right| \\
& =: T_{11}+T_{12} .
\end{aligned}
$$

To bound $T_{11}$ and $T_{12}$, let us define two sets of functions

$$
\begin{aligned}
& \mathcal{G}_{1}:=\left\{g: g=u^{2} \text { where } u \in \mathcal{F}\right\}, \\
& \mathcal{G}_{2}:=\left\{g: g=|\nabla u|^{2}+V|u|^{2} \text { where } u \in \mathcal{F}\right\} .
\end{aligned}
$$

We assume that the set $\mathcal{F}$ satisfies $\sup _{u \in \mathcal{F}}\|u\|_{L^{\infty}} \leq M_{\mathcal{F}}<\infty$ so that $\sup _{g \in \mathcal{G}_{1}}\|g\|_{L^{\infty}} \leq$ $M_{\mathcal{F}}^{2}$. Assume further that $\sup _{g \in \mathcal{G}_{2}}\|g\|_{L^{\infty}} \leq M_{\mathcal{G}_{2}}<\infty$. Now let us first derive a high-probability lower bound for $\mathcal{E}_{n, 2}\left(u_{n}\right)$. For doing so, we define for $n \in \mathbb{N}$ and $\delta>0$ the constant

$$
\xi_{1}(n, \delta):=2 R_{n}\left(\mathcal{G}_{1}\right)+4 M_{\mathcal{F}}^{2} \cdot \sqrt{\frac{2 \ln (4 / \delta)}{n}},
$$

where $R_{n}\left(\mathcal{G}_{1}\right)$ denotes the Rademacher complexity of the set $\mathcal{G}_{1}$. We also define the event

$$
A_{1}(n, \delta):=\left\{\left|\mathcal{E}_{n, 2}\left(u_{n}\right)-\mathcal{E}_{2}\left(u_{n}\right)\right| \leq \xi_{1}(n, \delta)\right\} .
$$

Then applying Lemma 4.3 to $\mathcal{G}_{1}$ we have that

$$
\mathbf{P}\left[A_{1}(n, \delta)\right] \geq 1-\delta .
$$

Since by assumption $\mathcal{E}_{2}\left(u_{n}\right)=1$, within the event $A_{1}(n, \delta)$ we have $\mathcal{E}_{n, 2}\left(u_{n}\right) \geq$ $1-\xi_{1}(n, \delta)$ and hence

$$
\mathbf{P}\left[\mathcal{E}_{n, 2}\left(u_{n}\right) \geq 1-\xi_{1}(n, \delta)\right] \geq 1-\delta .
$$

Notice that $\mathcal{E}_{V}\left(u_{n}\right) \leq M_{\mathcal{G}_{2}}$ by the assumption on $\mathcal{G}_{2}$. Therefore if $\xi_{1}(n, d)<1$, then

$$
\mathbf{P}\left[T_{12} \leq \frac{M_{\mathcal{G}_{2}} \cdot \xi_{1}(n, \delta)}{1-\xi_{1}(n, \delta)}\right] \geq \mathbf{P}\left[A_{1}(n, \delta)\right] \geq 1-\delta .
$$


Next to bound $T_{11}$, let us define the constant

$$
\xi_{2}(n, \delta):=2 R_{n}\left(\mathcal{G}_{2}\right)+4 M_{\mathcal{G}_{2}} \cdot \sqrt{\frac{2 \ln (4 / \delta)}{n}}
$$

and the event

$$
A_{2}(n, \delta):=\left\{\sup _{u \in \mathcal{F}}\left|\mathcal{E}_{n, V}(u)-\mathcal{E}_{V}(u)\right| \leq \xi_{2}(n, \delta)\right\} .
$$

Then applying again Lemma 4.3 to $\mathcal{G}_{2}$ leads to

$$
\mathbf{P}\left[A_{2}(n, \delta)\right] \geq 1-\delta
$$

As a result of (4.5) and (4.8), one has that if $\xi_{1}(n, d)<1$ then

$$
\mathbf{P}\left[T_{11} \leq \frac{\xi_{2}(n, d)}{1-\xi_{1}(n, d)}\right] \geq \mathbf{P}\left[A_{1}(n, \delta) \cap A_{2}(n, \delta)\right] \geq 1-2 \delta .
$$

Therefore it follows from (4.6) and (4.9) that

$$
\mathbf{P}\left[T_{1} \leq \frac{M_{\mathcal{G}_{2}} \cdot \xi_{1}(n, d)+\xi_{2}(n, d)}{1-\xi_{1}(n, d)}\right] \geq \mathbf{P}\left[A_{1}(n, \delta) \cap A_{2}(n, \delta)\right] \geq 1-2 \delta .
$$

Bounding $T_{2}$. Similar to the process of bounding $T_{1}$, by assuming $\left\|u_{\mathcal{F}}\right\|_{L^{2}}=1$ we first bound $T_{2}$ as follows

$$
\begin{aligned}
T_{2} & \leq\left|\frac{\mathcal{E}_{n, V}\left(u_{\mathcal{F}}\right)}{\mathcal{E}_{n, 2}\left(u_{\mathcal{F}}\right)}-\frac{\mathcal{E}_{V}\left(u_{\mathcal{F}}\right)}{\mathcal{E}_{2}\left(u_{\mathcal{F}}\right)}\right| \\
& \leq \frac{\left|\mathcal{E}_{n, V}\left(u_{\mathcal{F}}\right)-\mathcal{E}_{V}\left(u_{\mathcal{F}}\right)\right|}{\mathcal{E}_{n, 2}\left(u_{\mathcal{F}}\right)}+\frac{\mathcal{E}_{V}\left(u_{\mathcal{F}}\right)}{\mathcal{E}_{2}\left(u_{\mathcal{F}}\right) \cdot \mathcal{E}_{n, 2}\left(u_{\mathcal{F}}\right)}\left|\mathcal{E}_{2}\left(u_{\mathcal{F}}\right)-\mathcal{E}_{n, 2}\left(u_{\mathcal{F}}\right)\right| \\
& =: T_{21}+T_{22} .
\end{aligned}
$$

Since $u_{\mathcal{F}}$ does not depend on the sample points $\left\{X_{i}\right\}$, applying Hoeffding's inequality from Lemma 4.2 yields that

$$
\mathbf{P}\left[A_{3}(n, \delta)\right]:=\mathbf{P}\left[\left|\mathcal{E}_{n, 2}\left(u_{\mathcal{F}}\right)-\mathcal{E}_{2}\left(u_{\mathcal{F}}\right)\right| \leq \xi_{3}(n, \delta)\right] \geq 1-\delta,
$$

where

$$
\xi_{3}(n, \delta):=M_{\mathcal{F}}^{2} \cdot \sqrt{\frac{\ln (2 / \delta)}{2 n}} .
$$

Since by assumption $\mathcal{E}_{2}\left(u_{\mathcal{F}}\right)=1$, this implies further that

$$
\mathbf{P}\left[\mathcal{E}_{n, 2}\left(u_{\mathcal{F}}\right) \geq 1-\xi_{3}(n, \delta)\right] \geq \mathbf{P}\left[A_{3}(n, \delta)\right] \geq 1-\delta .
$$

Combining (4.11) and (4.13) implies that if $\xi_{3}(n, \delta)<1$, then

$$
\mathbf{P}\left[T_{22} \leq \frac{M_{\mathcal{G}_{2}} \cdot \xi_{3}(n, \delta)}{1-\xi_{3}(n, \delta)}\right] \geq \mathbf{P}\left[A_{3}(n, \delta)\right] \geq 1-\delta .
$$

In addition, as a consequence of (4.13) and (4.8), we have

$$
\mathbf{P}\left[T_{21} \leq \frac{\xi_{2}(n, \delta)}{1-\xi_{3}(n, \delta)}\right] \geq \mathbf{P}\left[A_{2}(n, \delta) \cap A_{3}(n, \delta)\right] \geq 1-2 \delta .
$$

Therefore it holds that

$$
\mathbf{P}\left[T_{2} \leq \frac{\xi_{2}(n, \delta)+M_{\mathcal{G}_{2}} \cdot \xi_{3}(n, \delta)}{1-\xi_{3}(n, \delta)}\right] \geq 1-2 \delta .
$$


Lemma 4.2 (Hoeffding). Let $Z_{1}, Z_{2}, \cdots, Z_{n}$ be i.i.d. random variables with $a_{i} \leq$ $Z_{i} \leq b_{i}$ a.s. Then for any $t>0$,

$$
\mathbf{P}\left(\left|\frac{\sum_{i=1}^{n} Z_{i}}{n}-\mathbf{E} Z\right| \geq t\right) \leq 2 \exp \left(-\frac{2 n^{2} t^{2}}{\sum_{i=1}^{n}\left(b_{i}-a_{i}\right)^{2}}\right) .
$$

We recall the following useful PAC-type generalization bound via the Rademacher complexity.

Lemma 4.3. [30, Theorem 26.5] Let $Z_{1}, Z_{2}, \cdots, Z_{n}$ be i.i.d. random variables. Let $\mathcal{G}$ be a function class such that $\sup _{g \in \mathcal{G}}\|g\|_{L^{\infty}(\Omega)} \leq C_{\mathcal{G}}$ and that $\mathcal{G}$ is symmetric, i.e. $\mathcal{G}=-\mathcal{G}$. Then with probability at least $1-\delta$,

$$
\sup _{g \in \mathcal{G}}\left|\frac{1}{n} \sum_{i=1}^{n} g\left(Z_{i}\right)-\mathbf{E} g(Z)\right| \leq 2 R_{n}(\mathcal{G})+4 C_{\mathcal{G}} \sqrt{\frac{2 \ln (4 / \delta)}{n}} .
$$

Combining the bounds derived above leads to the following oracle inequality. Recall the quantities $\xi_{i}(n, \delta), i=1,2,3$ defined in (4.3), (4.7) and (4.12).

Theorem 4.4. Let $u_{n}=\arg \min _{u \in \mathcal{F}} \mathcal{E}_{n}(u)$. Let $\delta \in\left(0, \frac{1}{3}\right)$ be fixed. Assume that $\xi_{i}(n, \delta)<1, i=1,2,3$.

Then with probability at least $1-3 \delta$,

$$
\begin{aligned}
\mathcal{E}\left(u_{n}\right)-\mathcal{E}\left(u^{*}\right) & \leq \frac{M_{\mathcal{G}_{2}} \cdot \xi_{1}(n, d)+\xi_{2}(n, d)}{1-\xi_{1}(n, d)}+\frac{M_{\mathcal{G}_{2}} \cdot \xi_{3}(n, \delta)+\xi_{2}(n, \delta)}{1-\xi_{3}(n, \delta)} \\
& +\left(\inf _{u \in \mathcal{F}} \mathcal{E}(u)-\mathcal{E}\left(u^{*}\right)\right) .
\end{aligned}
$$

4.2. Bounding the Approximation Error. Recall the spectral Barron space $\mathcal{B}^{s}(\Omega)$ defined in (2.4) and the set of two-layer networks $\mathcal{F}_{\mathrm{SP}_{\tau}, m}(B)$ defined by setting $\phi=\mathrm{SP}_{\tau}$ in (2.5). The following theorem bounds the approximation error (the third term) in (4.15) when $u^{*} \in \mathcal{B}(\Omega)$ and $\mathcal{F}=\mathcal{F}_{\mathrm{SP}_{\tau}, m}$.

Theorem 4.5. Let the ground state $u^{*} \in \mathcal{B}(\Omega)$ with $\left\|u^{*}\right\|_{L^{2}(\Omega)}=1$. Let $u_{m} \in$ $\mathcal{F}_{\mathrm{SP}_{\tau}, m}\left(\|u\|_{\mathcal{B}(\Omega)}\right)$ be defined in Lemma 2.2. Assume that $V$ satisfies Assumption 2.1. Assume in addition that

$$
\eta\left(\left\|u^{*}\right\|_{\mathcal{B}(\Omega)}, m\right):=\frac{\left\|u^{*}\right\|_{\mathcal{B}(\Omega)} \cdot(6 \ln m+30)}{\sqrt{m}} \leq \frac{1}{2} .
$$

Then

$$
\mathcal{E}\left(u_{m}\right)-\mathcal{E}\left(u^{*}\right) \leq\left(2\left(1+V_{\max }\right)\left(\sqrt{\frac{\lambda^{*}}{\min \left(1, V_{\min }\right)}}+1\right)+3 \lambda^{*}\right) \eta\left(\left\|u^{*}\right\|_{\mathcal{B}(\Omega)}, m\right)
$$

Proof. By assumption $\mathcal{E}_{2}\left(u^{*}\right)=\left\|u^{*}\right\|_{L^{2}(\Omega)}^{2}=1$. Then $\mathcal{E}\left(u^{*}\right)=\mathcal{E}_{V}\left(u^{*}\right)=\lambda^{*}$. Since $V \geq V_{\min }>0$, this implies that

$$
\left\|u^{*}\right\|_{H^{1}(\Omega)}^{2} \leq \frac{\mathcal{E}_{V}\left(u^{*}\right)}{\min \left(1, V_{\min }\right)}=\frac{\lambda^{*}}{\min \left(1, V_{\min }\right)} .
$$

Now observe that

$$
\begin{aligned}
\mathcal{E}\left(u_{m}\right)-\mathcal{E}\left(u^{*}\right) & =\frac{\mathcal{E}_{V}\left(u_{m}\right)}{\mathcal{E}_{2}\left(u_{m}\right)}-\frac{\mathcal{E}_{V}\left(u^{*}\right)}{\mathcal{E}_{2}\left(u^{*}\right)} \\
& =\frac{\mathcal{E}_{V}\left(u_{m}\right)-\mathcal{E}_{V}\left(u^{*}\right)}{\mathcal{E}_{2}\left(u_{m}\right)}+\frac{\mathcal{E}_{2}\left(u^{*}\right)-\mathcal{E}_{2}\left(u_{m}\right)}{\mathcal{E}_{2}\left(u_{m}\right)} \cdot \mathcal{E}\left(u^{*}\right) .
\end{aligned}
$$


Thanks to Lemma 2.2. $\left\|u_{m}-u^{*}\right\|_{H^{1}(\Omega)} \leq \eta\left(\left\|u^{*}\right\|_{\mathcal{B}(\Omega)}, m\right)<1$. This implies that

$$
1-\eta\left(\left\|u^{*}\right\|_{\mathcal{B}(\Omega)}, m\right) \leq\left\|u_{m}\right\|_{L^{2}(\Omega)} \leq 1+\eta\left(\left\|u^{*}\right\|_{\mathcal{B}(\Omega)}, m\right)
$$

and that

$$
\begin{aligned}
\left|\mathcal{E}_{2}\left(u_{m}\right)-\mathcal{E}_{2}\left(u^{*}\right)\right| & =\left(\left\|u^{*}\right\|_{L^{2}(\Omega)}+\left\|u_{m}\right\|_{L^{2}(\Omega)}\right)\left|\left\|u^{*}\right\|_{L^{2}(\Omega)}-\left\|u_{m}\right\|_{L^{2}(\Omega)}\right| \\
& \leq\left(2+\eta\left(\left\|u^{*}\right\|_{\mathcal{B}(\Omega)}, m\right)\right) \eta\left(\left\|u^{*}\right\|_{\mathcal{B}(\Omega)}, m\right) \\
& \leq 3 \eta\left(\left\|u^{*}\right\|_{\mathcal{B}(\Omega)}, m\right) .
\end{aligned}
$$

In addition, it follows from the boundedness of $V$ that

$$
\begin{aligned}
\left|\mathcal{E}_{V}\left(u_{m}\right)-\mathcal{E}_{V}\left(u^{*}\right)\right| & \leq\left(1+V_{\max }\right)\left(\left\|u^{*}\right\|_{H^{1}(\Omega)}+\left\|u_{m}\right\|_{H^{1}(\Omega)}\right)\left\|u^{*}-u_{m}\right\|_{H^{1}(\Omega)} \\
& \leq\left(1+V_{\max }\right)\left(2\left\|u^{*}\right\|_{H^{1}(\Omega)}+\eta\left(\left\|u^{*}\right\|_{\mathcal{B}(\Omega)}, m\right)\right) \eta\left(\left\|u^{*}\right\|_{\mathcal{B}(\Omega)}, m\right) \\
& \leq\left(1+V_{\max }\right)\left(\sqrt{\frac{\lambda^{*}}{\min \left(1, V_{\min }\right)}}+1\right) \eta\left(\left\|u^{*}\right\|_{\mathcal{B}(\Omega)}, m\right),
\end{aligned}
$$

where we have used (4.18) in the last inequality. Finally, the estimate follows simply by substituting the last two estimates into (4.19).

4.3. Bounding the Statistical Error. In this section we proceed to bound the statistical errors (the first two terms on the right side of (4.15)). This is achieved by controlling the Rademacher complexities of the function classes $\mathcal{G}_{1}$ and $\mathcal{G}_{2}$ defined in (4.20). More specifically, since we set the trial functions $\mathcal{F}=\mathcal{F}_{\mathrm{SP}_{\tau}, m}$, we need to bound the Rademacher complexities of the following

$$
\begin{aligned}
& \mathcal{G}_{\mathrm{SP}_{\tau}, m, 1}(B):=\left\{g=u^{2}: u \in \mathcal{F}_{\mathrm{SP}_{\tau}, m}(B)\right\}, \\
& \mathcal{G}_{\mathrm{SP}_{\tau}, m, 2}(B):=\left\{g=|\nabla u|^{2}+V|u|^{2}: u \in \mathcal{F}_{\mathrm{SP}_{\tau}, m}(B)\right\} .
\end{aligned}
$$

Theorem 4.6. Assume that $\|V\|_{L^{\infty}(\Omega)} \leq V_{\max }$. consider the sets $\mathcal{G}_{\mathrm{SP}_{\tau}, m, 1}(B)$ and $\mathcal{G}_{\mathrm{SP}_{\tau}, m, 2}(B)$ with $\tau=\sqrt{m}$ and $B>0$. Then there exist positive constants $C_{1}(B, d, F)$ and $C_{2}\left(B, d, V_{\max }\right)$ depending polynomially on $B, d, V_{\max }$ such that

$$
\begin{aligned}
& R_{n}\left(\mathcal{G}_{\mathrm{SP}_{\tau}, m, 1}(B)\right) \leq \frac{C_{1}(B, d) \sqrt{m}(\sqrt{\ln m}+1)}{\sqrt{n}}, \\
& R_{n}\left(\mathcal{G}_{\mathrm{SP}_{\tau}, m, 2}(B)\right) \leq \frac{C_{2}\left(B, d, V_{\max }\right) \sqrt{m}(\sqrt{\ln m}+1)}{\sqrt{n}} .
\end{aligned}
$$

To prove Theorem 4.6, we rely on the celebrated Dudley's theorem [9], which bounds the Rademacher complexities in terms of the metric entropy. Below we restate the Dudley's theorem by following [36, Theorem 1.19].

Theorem 4.7 (Dudley's theorem [36, Theorem 1.19]). Let $\mathcal{F}$ be a function class such that $\sup _{f \in \mathcal{F}}\|f\|_{\infty} \leq M$. Then the Rademacher complexity $R_{n}(\mathcal{F})$ satisfies that

$$
R_{n}(\mathcal{F}) \leq \inf _{0 \leq \delta \leq M}\left\{4 \delta+\frac{12}{\sqrt{n}} \int_{\delta}^{M} \sqrt{\ln \mathcal{N}\left(\varepsilon, \mathcal{F},\|\cdot\|_{\infty}\right)} d \varepsilon\right\}
$$

where $\mathcal{N}\left(\varepsilon, \mathcal{F},\|\cdot\|_{\infty}\right)$ denotes the $\varepsilon$-covering number of $\mathcal{F}$ w.r.t the $L_{\infty}$-norm.

In order to apply Dudley's theorem, we need to bound the $\delta$-covering numbers of the sets $\mathcal{G}_{\mathrm{SP}_{\tau}, m, i}(B), i=1,2$. To this end, it will be convenient to introduce the following functions

$$
\mathcal{M}(\delta, \Lambda, m, d):=\frac{4 B \Lambda}{\delta} \cdot\left(\frac{12 B \Lambda}{\delta}\right)^{m} \cdot\left(\frac{3 \Lambda}{\delta}\right)^{d m} \cdot\left(\frac{3 \Lambda}{\delta}\right)^{m}
$$




$$
\begin{aligned}
\mathcal{Z}(M, \Lambda, d):= & M\left(\sqrt{(\ln (4 B \Lambda))_{+}}+\sqrt{(\ln (12 B \Lambda)+d \ln (3 \Lambda)+\ln (3 \Lambda))_{+}}\right) \\
& +\sqrt{d+3} \int_{0}^{M} \sqrt{(\ln (1 / \varepsilon))_{+}} d \varepsilon .
\end{aligned}
$$

Lemma 4.8. Consider the two sets $\mathcal{G}_{\mathrm{SP}_{\tau}, m, i}(B), i=1,2$ defined in (4.20) with $B>0$. Then for any $\delta>0$,

$$
\mathcal{N}\left(\delta, \mathcal{G}_{\mathrm{SP}_{\tau}, m, i}(B),\|\cdot\|_{\infty}\right) \leq \mathcal{M}\left(\delta, \Lambda_{i}, m, d\right), \quad i=1,2,
$$

where the constants $\Lambda_{i}, i=1,2$ satisfy that

$$
\Lambda_{1} \leq 36 B(5+8 B), \quad \Lambda_{2} \leq 64 B^{2} \sqrt{m}+8 B+36 V_{\max } B(5+8 B) .
$$

Proof. The proof follows directly from [24, Lemma 5.5] and [24, Lemma 5.7] by slightly adjusting the constants. We thus omit the details.

Proof of Theorem 4.6. First from the definition of $\mathcal{F}_{\mathrm{SP}_{\tau}, m}(B)$ and the fact that $\left\|\mathrm{SP}_{\tau}\right\|_{W^{1, \infty}(\Omega)} \leq 3+\frac{1}{\tau}$ (see [24, Lemma 4.6]), one has the following uniform bound

$$
\sup _{u \in \mathcal{F}_{\mathrm{SP}_{\tau}, m}(B)}\|u\|_{W^{1, \infty}(\Omega)} \leq 2 B+4 B\left\|\mathrm{SP}_{\tau}\right\|_{W^{1, \infty}(\Omega)} \leq 16 B .
$$

This implies that

$$
\begin{aligned}
& \sup _{f \in \mathcal{F}_{\mathrm{SP}_{\tau}, m}(B)}\|f\|_{L^{\infty}(\Omega)} \leq 16 B=M_{\mathcal{F}}, \\
& \sup _{g \in \mathcal{G}_{\mathrm{SP}_{\tau}, m, 1}(B)}\|g\|_{L^{\infty}(\Omega)} \leq(16 B)^{2}=: M_{1}, \\
& \sup _{g \in \mathcal{G}_{\mathrm{SP}_{\tau}, m, 2}(B)}\|g\|_{L^{\infty}(\Omega)} \leq\left(1+V_{\max }\right)(16 B)^{2}=: M_{2} .
\end{aligned}
$$

Applying Theorem 4.7 with $\delta=0$, we obtain from Lemma 4.8 and the estimates in the last line that

$$
R_{n}\left(\mathcal{G}_{\mathrm{SP}_{\tau}, m, i}(B)\right) \leq \mathcal{Z}\left(M_{i}, \Lambda_{i}, d\right) \sqrt{\frac{m}{n}} .
$$

Moreover, after plugging the bounds on $\Lambda_{i}$ (see (4.26) $)$ into $\mathcal{Z}\left(M_{i}, \Lambda_{i}, d\right)$, it is easy to see that

$$
\begin{aligned}
& \mathcal{Z}\left(M_{1}, \Lambda_{1}, d\right) \leq C_{1}(B, d), \\
& \mathcal{Z}\left(M_{1}, \Lambda_{1}, d\right) \leq C_{2}\left(B, d, V_{\max }\right) \sqrt{\ln m},
\end{aligned}
$$

where the positive constants $C_{1}(B, d)$. and $C_{2}\left(B, d, V_{\max }\right)$ depend on $B$ and $d$ polynomially. Combining the estimates finishes the proof of Theorem 4.6 .

4.4. Proof of Theorem 2.3. The proof follows directly by combining Theorem 4.4. Theorem 4.5 and Theorem 4.6.

\section{Regularity of the Ground State of Schrödinger Operator in the spectral Barron Space (Proof of Theorem 2.5)}

In this section we aim to prove the regularity of the ground state $u^{*}$ in the spectral Barron space as shown in Theorem 2.5. Since our proof relies heavily on the spectrum theory of positive linear operators on ordered Banach spaces (especially the Krein-Rutman Theorem), we first recall some relevant terminologies and useful facts from linear functional analysis. 
5.1. A simple lemma on spectral radius. Let $E$ be a Banach space. Given a bounded linear operator $T$ on $E$, we recall the resolvent set of $T$ defined by

$$
\rho_{E}(T):=\{\lambda \in \mathbb{C} \mid(\lambda I-T) \text { is bijective on } E\} .
$$

The spectrum $\sigma_{E}(T)$ of $T$ is the set $\mathbb{C} \backslash \rho_{E}(T)$, where we have used the subscript $E$ to indicate the dependence on the Banach space $E$. We further denote by $\sigma_{p, E}(T)$ the point spectrum of $T$, i.e.

$\sigma_{p, E}(T)=\{\lambda \in \mathbb{C} \mid$ there exists $v \in E$ and $v \neq 0$ such that $(\lambda I-T) v=0\} \subset \sigma_{E}(T)$,

where we call $\lambda \in \sigma_{p, E}(T)$ an eigenvalue of $T$ and $v$ an eigenvector of $T$. The spectral radius of $T$ is given by

$$
r_{E}(T):=\sup \left\{|\lambda| \mid \lambda \in \sigma_{E}(T)\right\} .
$$

Recall the Beurling-Gelfand's formula $r_{E}(T)=\lim _{n \rightarrow \infty}\left\|T^{n}\right\|^{1 / n}$. We also note that if $T: E \rightarrow E$ is compact, then $\sigma_{E}(T) \backslash\{0\}=\sigma_{p, E}(T) \backslash\{0\}$.

Lemma 5.1. Let $T: E \rightarrow E$ be a linear compact operator on a Hilbert space $E$ equipped with an inner product $(\cdot, \cdot)_{E}$ and the associated norm $\|\cdot\|_{E}$. Let $F \varsubsetneqq E$ be a dense subspace of $E$. Assume that $F$ is a Banach space equipped with the norm $\|\cdot\|_{F}$ and that $T: F \rightarrow F$ is also compact. Then $r_{E}(T)=r_{F}(T)$.

Proof. First thanks to $F \subset E$, we claim that $r_{E}(T) \geq r_{F}(T)$. In fact, if $r_{F}(T)=0$, then this holds trivially. In addition, if $r_{E}(T)=0$, then $r_{F}(T)=0$. Indeed, if assume otherwise that $r_{F}(T)>0$, then since $\sigma_{E}(T) \backslash\{0\}=\sigma_{p, E}(T) \backslash\{0\}$ due to the compactness of $T$, there exists $\lambda \neq 0$ and $v \in F \backslash\{0\}$ such that $(\lambda I-T) v=0$. Since $F \subset E$, we have $v \in E$ and hence $\lambda \in \sigma_{E}(T)$. This contradicts with the assumption that $r_{E}(T)=0$ and thus proves $r_{F}(T)=0$. Finally, assume that both $r_{E}(T)$ and $r_{F}(T)$ are positive. We claim that $\sigma_{F}(T) \backslash\{0\} \subset \sigma_{E}(T) \backslash\{0\}$ which immediately implies that $r_{E}(T) \geq r_{F}(T)$. In fact, for any $\lambda \in \sigma_{F}(T) \backslash\{0\}=\sigma_{p, F}(T) \backslash\{0\}$, there exists $v \in F \backslash\{0\}$ such that $(\lambda I-T) v=0$. Since $F \subset E$, we have $\lambda \in \sigma_{E}(T) \backslash\{0\}$.

Next we show that $r_{E}(T)=r_{F}(T)$. Assume otherwise that $r_{E}(T)>r_{F}(T)$. By the definition of $r_{E}(T)$ and the assumption that $T: E \rightarrow E$ is a compact operator, there exists an eigenvalue $\lambda \in \rho_{p, E}(T)$ such that $r_{E}(T) \geq|\lambda|>r_{F}(T)$. This implies that $\operatorname{Ker}_{F}(\lambda I-T):=\{u \in F:(\lambda I-T) u=0\}=\{0\}$, i.e., $\operatorname{Ker}_{E}(\lambda I-T) \cap F=\{0\}$. Since $F$ is a dense subset of $E$, this implies that $\operatorname{Ker}_{E}(\lambda I-T) \subset \bar{F}^{\perp}=E^{\perp}=\{0\}$. This contradicts with the fact that $\lambda$ is an eigenvalue of $T$ on $E$ and completes the proof of the lemma.

5.2. Krein-Rutman theorem and the leading eigenvalue. In this section, we recall the famous Krein-Rutman Theorem [22] on the leading eigenvalue and eigenfunction of positive operators on ordered Banach spaces. To this end, let us first recall some terminologies on ordered Banach spaces. Given a Banach space $E$, a closed convex subset $K \subset E$ is called a cone on $E$ if $\alpha K \subset K$ for every $\alpha>0$ and $K \cap\{-K\}=\{0\}$. A cone $K$ induces a natural partial ordering $\leq$ on the Banach space $E: x \leq y$ if and only if $y-x \in K$. Therefore a Banach space $E$ with a cone $K$ is called an ordered Banach space, denoted by $(E, K)$. If the cone $K$ satisfies that $\overline{K-K}=E$, then the cone $K$ is called a total cone. We define $\dot{K}=K \backslash\{0\}$ and denote by $K^{\circ}$ the interior of $K$. If $K$ has nonempty interior $K^{\circ}$, then $K$ is called a solid cone. It is not hard to see that a solid cone is total. 
Example 5.1. Consider the Banach space $C(\bar{\Omega})$ of continuous functions on a bounded domain $\Omega \subset \mathbb{R}^{d}$. The space $C(\bar{\Omega})$ is an ordered Banach space with cone $C_{+}(\bar{\Omega})$ consisting of nonnegative functions in $C(\bar{\Omega})$. This cone is solid since any strictly positive function is an interior point.

Consider two ordered Banach spaces $E$ and $F$, with cones $P$ and $Q$ respectively. A linear operator $T: E \rightarrow F$ is called positive if $T(P) \subset Q$, and strictly positive if $T(\dot{P}) \subset \dot{Q}$. If in addition $K$ is solid, then $T$ is called strongly positive if $T(\dot{P}) \subset Q^{\circ}$.

Theorem 5.2 (Krein-Rutman). Let $E$ be an ordered Banach space with a total cone $K \subset E$. Let $T: E \rightarrow E$ be a linear compact positive operator with spectral radius $r(T)>0$. Then $r(T)$ is an eigenvalue of $T$ and of the dual $T^{*}$ with corresponding eigenvectors $u \in K \backslash\{0\}$ and $u^{*} \in K^{*} \backslash\{0\}$.

As an important consequence of the Krein-Rutman theorem, the following theorem establishes the simplicity of the leading eigenvalue of a strongly positive compact operator on an ordered Banach space.

Theorem 5.3 ([1, Theorem 3.2]). Let $E$ be an ordered Banach space with a solid cone $K$. Let $T: E \rightarrow E$ be a strongly positive compact operator. Then

(i) The spectral radius $r(T)>0$;

(ii) $r(T)$ is a simple eigenvalue with an eigenvector $u \in K^{\circ}$ and there is no other eigenvalue with a positive eigenvector.

5.3. Regularity of Ground State. Consider the Schrödinger operator $\mathcal{H}=-\Delta+$ $V$. Since $\inf _{x} V(x) \geq V_{\min }>0$, the standard Sobolev estimate implies that the inverse of $\mathcal{H}$ (with respect to the Neumann boundary condition), denoted by $\mathcal{S}:=$ $\mathcal{H}^{-1}$, is bounded from $L^{2}(\Omega)$ to $H^{2}(\Omega)$ and hence compact on $L^{2}(\Omega)$. Moreover, $\mathcal{S}$ has countable many eigenvalues $\left\{\mu_{j}\right\}_{j=0}^{\infty}$ with $\mu_{j} \downarrow 0$ as $j \rightarrow \infty$ and with $r(\mathcal{S})=$ $\mu_{0}=\frac{1}{\lambda_{0}}$.

Recall the spectral Barron space $\mathcal{B}^{s}(\Omega)$ defined in (2.4). We also recall from [24] the next important lemma which shows that the operator $\mathcal{S}: \mathcal{B}^{s}(\Omega) \rightarrow \mathcal{B}^{s+2}(\Omega)$ is bounded.

Lemma $5.4\left(\left[24\right.\right.$, Theorem 6]). Assume that $V \in \mathcal{B}^{s}(\Omega)$ with $s \geq 0$ and $\inf _{x \in \Omega} \geq$ $V_{\min }>0$. Then the operator $\mathcal{S}: \mathcal{B}^{s}(\Omega) \rightarrow \mathcal{B}^{s+2}(\Omega)$ is bounded.

Notice that the inclusion $\mathcal{I}: \mathcal{B}^{s+2}(\Omega) \hookrightarrow \mathcal{B}^{s}(\Omega)$ is compact. In fact, by definition the space $\mathcal{B}^{s}(\Omega)$ can be viewed as a weighted $\ell^{1}$ space $\ell_{W_{s}}^{1}\left(\mathbb{N}_{0}^{d}\right)$ of the cosine coefficients defined on the lattice $\mathbb{N}_{0}^{d}$ with the weight $W_{s}(k)=\left(1+\pi^{s}|k|_{1}^{s}\right)$. Therefore the inclusion satisfies that

$$
\|\mathcal{I} u\|_{\mathcal{B}^{s}(\Omega)}=\sum_{k \in \mathbb{N}_{0}^{d}} W_{s}(k)|\hat{u}(k)|=\sum_{k \in \mathbb{N}_{0}^{d}} \frac{W_{s}(k)}{W_{s+2}(k)} W_{s+2}(k)|\hat{u}(k)| .
$$

Since $\frac{W_{s}(k)}{W_{s+2}(k)} \rightarrow 0$ as $|k| \rightarrow \infty$, by a similar argument as used in the proof of 24 , Lemma 7.2], one can conclude that $\mathcal{I}$ is compact from $\ell_{W_{s+2}}^{1}\left(\mathbb{N}_{0}^{d}\right)$ to $\ell_{W_{s}}^{1}\left(\mathbb{N}_{0}^{d}\right)$ and hence from $\mathcal{B}^{s+2}(\Omega)$ to $\mathcal{B}^{s}(\Omega)$. The following corollary is then a direct consequence of Lemma 5.4 and compactness of the inclusion $\mathcal{I}$ from $\mathcal{B}^{s+2}(\Omega)$ to $\mathcal{B}^{s}(\Omega)$.

Corollary 5.5. Under the same assumption of Lemma 5.4, the operator $\mathcal{S}$ : $\mathcal{B}^{s}(\Omega) \rightarrow \mathcal{B}^{s}(\Omega)$ is compact. 
Note that by definition, we have $\mathcal{B}^{s}(\Omega) \hookrightarrow C(\bar{\Omega})$. Let us define the cone $\mathcal{B}_{+}^{s}(\Omega)$ of $\mathcal{B}^{s}(\Omega)$ by setting

$$
\mathcal{B}_{+}^{s}(\Omega):=\left\{f \in \mathcal{B}^{s}(\Omega): f \geq 0\right\} .
$$

Lemma 5.6. The operator $\mathcal{S}: \mathcal{B}^{s}(\Omega) \rightarrow \mathcal{B}^{s}(\Omega)$ is strongly positive, i.e. for any non-zero $f \in \mathcal{B}_{+}^{s}(\Omega)$, we have that $\mathcal{S} f(x)>0$ for all $x \in \bar{\Omega}$.

Proof. Let $f \in \mathcal{B}_{+}^{s}(\Omega) \subset C(\bar{\Omega})$. For any fixed $t>0$ and $x \in \Omega$, it follows by the Lie-Trotter splitting that

$$
e^{-t \mathcal{H}} f(x)=\lim _{n \rightarrow \infty}\left[e^{-\frac{t}{n} V} e^{\frac{t}{n} \Delta}\right]^{n} f(x) .
$$

Since $0<V_{\min } \leq V \leq V_{\max }<\infty$, we have $e^{-s V_{\max }} g \leq e^{-s V} g \leq e^{-s V_{\min }} g$ for any non-negative $g \in L^{\infty}(\Omega)$. Thanks to the fact that the heat semigroup $e^{t \Delta}$ is positivity-preserving, this implies that

$$
e^{-t V_{\max }} e^{t \Delta} f(x) \leq e^{-t \mathcal{H}} f(x) \leq e^{-t V_{\min }} e^{t \Delta} f(x) .
$$

Moreover, as a result of the semigroup property, the solution operator $\mathcal{S}$ can be written as

$$
\mathcal{S} f(x)=\int_{0}^{\infty} e^{-t \mathcal{H}} f(x) d t .
$$

Note that owing to the upper bound of (5.1) the integral is finite. It follows from the last identity and the lower bound of (5.1) that

$$
\mathcal{S} f(x) \geq \int_{0}^{\infty} e^{-t V_{\max }} e^{t \Delta} f(x) d t \geq \int_{1}^{2} e^{-t V_{\max }} e^{t \Delta} f(x) d t .
$$

Now since $f$ is non-negative, continuous and non-zero on $\Omega$, there exists a set $A \subset \Omega$ with $\operatorname{Leb}(A)>0$ and a constant $c>0$ such that $f \geq c>0$. Thanks to the Gaussian lower bound of the Neumann heat kernel estimate (see e.g. [4, Theorem 3.4]), there exist positive constants $c_{1}$ and $c_{2}$ such that

$$
\begin{aligned}
e^{t \Delta} f(x) & =\int_{\Omega} p_{t}(x, y) f(y) d y \\
& \geq \int_{\Omega} \frac{c_{1}}{t^{\frac{d}{2}}} e^{-\frac{|x-y|^{2}}{c_{2} t}} f(y) d y \\
& \geq \frac{c c_{1}}{t^{\frac{d}{2}}} e^{-\frac{\operatorname{diam}(\Omega)}{c_{2} t}} \operatorname{Leb}(A)>0 .
\end{aligned}
$$

Multiplying above with $e^{-t V_{\max }}$ and then integrating on $[1,2]$ with respect to $t$ yields that

$$
\mathcal{S} f(x) \geq c c_{1} \operatorname{Leb}(A) \int_{1}^{2} \frac{1}{t^{\frac{d}{2}}} e^{-\frac{\operatorname{diam}(\Omega)}{c_{2} t}} d t>0 .
$$
2.5 .

Now with the above preparations we are ready to present the proof of Theorem

Proof of Theorem 2.5. It is clear that the ground state $u^{*}$ of $\mathcal{H}$ is identical to the eigenfunction that corresponds to the spectral radius $r(\mathcal{S})=1 / \lambda^{*}$ of the inverse operator $\mathcal{S}=\mathcal{H}^{-1}$. In order to show that $u^{*} \in \mathcal{B}^{s+2}(\Omega)$, it suffices to show that $u^{*} \in \mathcal{B}^{s}(\Omega)$. In fact, since $\left(u^{*}, \lambda^{*}\right)$ solves the Neumann eigenvalue problem

$$
\mathcal{H} u^{*}=-\Delta u^{*}+V u^{*}=\lambda^{*} u^{*},
$$


we have $u^{*}=\lambda^{*} \mathcal{S} u^{*}$. An application of Lemma 5.4 implies that $u^{*} \in \mathcal{B}^{s+2}(\Omega)$ if and only if $u^{*} \in \mathcal{B}^{s}(\Omega)$. To show the latter, let us consider the operator $\mathcal{S}$ defined on the ordered Banach space $\mathcal{B}^{s}(\Omega)$ with the solid cone $\mathcal{B}_{+}^{s}(\Omega)$. Observe that $\mathcal{S}: L^{2}(\Omega) \rightarrow L^{2}(\Omega)$ is compact and that by Corollary $5.5 \mathcal{S}: \mathcal{B}^{s}(\Omega) \rightarrow \mathcal{B}^{s}(\Omega)$ is also compact. Therefore by Lemma 5.1 the spectral radii of $\mathcal{S}$ are identical when viewed as operators on $\mathcal{B}^{s}(\Omega)$ and $L^{2}(\Omega)$ respectively. It follows from Theorem 5.3 and the strongly positivity of $\mathcal{S}$ established in Lemma 5.6 that there exists a unique (up to a multiplicative constant) eigenfunction $u^{*} \in \mathcal{B}^{s}(\Omega)$ corresponding to the spectral radius $r(\mathcal{S})=1 / \lambda^{*}$. Moreover, $u^{*}$ is strictly positive on $\bar{\Omega}$. This completes the proof.

\section{REFERENCES}

[1] Herbert Amann. Fixed point equations and nonlinear eigenvalue problems in ordered banach spaces. SIAM review, 18(4):620-709, 1976.

[2] Francis Bach. Breaking the curse of dimensionality with convex neural networks. The Journal of Machine Learning Research, 18(1):629-681, 2017.

[3] Andrew R Barron. Universal approximation bounds for superpositions of a sigmoidal function. IEEE Transactions on Information theory, 39(3):930-945, 1993.

[4] Richard F Bass and Pei Hsu. Some potential theory for reflecting brownian motion in holder and lipschitz domains. The Annals of Probability, pages 486508, 1991.

[5] Z. Cai and J. Liu. Approximating quantum many-body wave functions using artificial neural networks. Phys. Rev. B, 97:035116, 2018.

[6] G. Carleo and M. Troyer. Solving the quantum many-body problem with artificial neural networks. Science, 355:602-606, 2017.

[7] Fan Chen, Jianguo Huang, Chunmei Wang, and Haizhao Yang. Friedrichs learning: Weak solutions of partial differential equations via deep learning. arXiv preprint arXiv:2012.08023, 2020.

[8] Kenny Choo, Antonio Mezzacapo, and Giuseppe Carleo. Fermionic neuralnetwork states for ab-initio electronic structure. Nature communications, 11(1):1-7, 2020.

[9] Richard M Dudley. The sizes of compact subsets of hilbert space and continuity of gaussian processes. Journal of Functional Analysis, 1(3):290-330, 1967.

[10] Weinan E, Chao Ma, Stephan Wojtowytsch, and Lei Wu. Towards a mathematical understanding of neural network-based machine learning: what we know and what we don't. arXiv preprint arXiv:2009.10713, 2020.

[11] Weinan E, Chao Ma, and Lei Wu. Barron spaces and the compositional function spaces for neural network models. arXiv preprint arXiv:1906.08039, 2019.

[12] Weinan E and Bing Yu. The deep ritz method: a deep learning-based numerical algorithm for solving variational problems. Communications in Mathematics and Statistics, 6(1):1-12, 2018.

[13] X. Gao and L.-M. Duan. Efficient representation of quantum many-body states with deep neural networks. Nat. Commun., 8:662, 2017.

[14] James Glimm and Arthur Jaffe. A functional integral point of view. Quantum Physics, 2nd edn. Springer, New York, 1987. 
[15] Yiqi Gu, Haizhao Yang, and Chao Zhou. Selectnet: Self-paced learning for high-dimensional partial differential equations. arXiv preprint arXiv:2001.04860, 2020.

[16] Jiequn Han, Arnulf Jentzen, and Weinan E. Solving high-dimensional partial differential equations using deep learning. Proceedings of the National Academy of Sciences, 115(34):8505-8510, 2018.

[17] Jiequn Han, Jianfeng Lu, and Mo Zhou. Solving high-dimensional eigenvalue problems using deep neural networks: A diffusion monte carlo like approach. Journal of Computational Physics, 423:109792, 2020.

[18] Jiequn Han, Linfeng Zhang, and Weinan E. Solving many-electron Schrödinger equation using deep neural networks. Journal of Computational Physics, 399:108929, 2019.

[19] Jan Hermann, Zeno Schätzle, and Frank Noé. Deep-neural-network solution of the electronic Schrödinger equation. Nature Chemistry, 12(10):891-897, 2020.

[20] Qingguo Hong, Jonathan W Siegel, and Jinchao Xu. Rademacher complexity and numerical quadrature analysis of stable neural networks with applications to numerical pdes. arXiv preprint arXiv:2104.02903, 2021.

[21] Jason M Klusowski and Andrew R Barron. Approximation by combinations of relu and squared relu ridge functions with $\ell^{1}$ and $\ell^{0}$ controls. IEEE Transactions on Information Theory, 64(12):7649-7656, 2018.

[22] Mark Grigor'evich Krein and Moisei Aronovich Rutman. Linear operators leaving invariant a cone in a Banach space. Amer. Math. Soc. Transl. Ser. I, 10:199-325, 1950.

[23] Yann LeCun, Yoshua Bengio, and Geoffrey Hinton. Deep learning. Nature, 521(7553):436-444, 2015.

[24] Jianfeng Lu, Yulong Lu, and Min Wang. A priori generalization analysis of the deep ritz method for solving high dimensional elliptic equations. arXiv preprint arXiv:2101.01708.

[25] Tao Luo and Haizhao Yang. Two-layer neural networks for partial differential equations: Optimization and generalization theory. arXiv preprint arXiv:2006.15733, 2020.

[26] Siddhartha Mishra and Roberto Molinaro. Estimates on the generalization error of physics informed neural networks (PINNs) for approximating PDEs, 2020. arXiv preprint arXiv:2006.16144.

[27] David Pfau, James S Spencer, Alexander GDG Matthews, and W Matthew C Foulkes. Ab initio solution of the many-electron schrödinger equation with deep neural networks. Physical Review Research, 2(3):033429, 2020.

[28] Maziar Raissi, Paris Perdikaris, and George E Karniadakis. Physics-informed neural networks: A deep learning framework for solving forward and inverse problems involving nonlinear partial differential equations. Journal of Computational Physics, 378:686-707, 2019.

[29] Jürgen Schmidhuber. Deep learning in neural networks: An overview. Neural networks, 61:85-117, 2015.

[30] Shai Shalev-Shwartz and Shai Ben-David. Understanding machine learning: From theory to algorithms. Cambridge university press, 2014.

[31] Yeonjong Shin, Jerome Darbon, and George Em Karniadakis. On the convergence of physics informed neural networks for linear second-order elliptic and parabolic type PDEs, 2020. arXiv preprint arXiv:2004.01806. 
[32] Yeonjong Shin, Zhongqiang Zhang, and George Em Karniadakis. Error estimates of residual minimization using neural networks for linear PDEs, 2020. arXiv preprint arXiv:2010.08019.

[33] Jonathan W Siegel and Jinchao Xu. Approximation rates for neural networks with general activation functions. Neural Networks, 2020.

[34] Jonathan W Siegel and Jinchao Xu. High-order approximation rates for neural networks with $\mathrm{ReLU}^{k}$ activation functions. arXiv preprint arXiv:2012.07205, 2020 .

[35] Justin Sirignano and Konstantinos Spiliopoulos. DGM: A deep learning algorithm for solving partial differential equations. Journal of computational physics, 375:1339-1364, 2018.

[36] Michael M. Wolf. Mathematical Founda-

tions of Supervised Learning, $2020 . \quad$ URL:
https://www-m5.ma.tum.de/foswiki/pub/M5/Allgemeines/MA4801_2020S/ML_notes_main.pdf. Last visited on $2020 / 12 / 5$.

[37] Jinchao Xu. Finite neuron method and convergence analysis. Communications in Computational Physics, 28(5):1707-1745, 2020.

[38] Yaohua Zang, Gang Bao, Xiaojing Ye, and Haomin Zhou. Weak adversarial networks for high-dimensional partial differential equations. Journal of Computational Physics, 411:109409, 2020.

(JL) Departments of Mathematics, Physics, and Chemistry, Duke University, Box 90320, Durham, NC 27708.

Email address: jianfeng@math.duke.edu

(YL) Department of Mathematics and Statistics, Lederle Graduate Research Tower, University of Massachusetts, 710 N. Pleasant Street, Amherst, MA 01003.

Email address: lu@math.umass.edu 